\title{
Urinary $\beta 2$-microglobulin and disease activity in patients with tubulointerstitial nephritis and uveitis syndrome
}

\author{
Lorraine M. Provencher ${ }^{1}$, Aaron M. Fairbanks ${ }^{1}$, Michael D. Abramoff ${ }^{1,2,3}$ and Nasreen A. Syed ${ }^{1,3,4^{*}}$ (D)
}

\begin{abstract}
Background: Urinary $\beta 2$-microglobulin (Uß2M) is elevated in tubulointerstitial nephritis and uveitis (TINU) syndrome and has emerged as an important diagnostic tool. This study aims to determine whether Uß2M correlates with uveitis activity in TINU.

Methods: Retrospective observational case series of nine patients with TINU and $\geq 30$ days follow-up. Presenting symptoms, visual acuity, uveitis characteristics, follow-up, U $2 \mathrm{M}$, serum creatinine ( $\mathrm{SCr}$ ), urinalysis, and renal biopsy results were collected.

Results: A correlation between U 2 M and anterior chamber (AC) cell ( $r=0.69,95 \% \mathrm{Cl} 0.46-0.84)$, flare $(r=0.65,95 \%$ Cl 0.39-0.81), trended toward a stronger correlation than SCr and AC cell $(r=0.59,95 \% \mathrm{Cl} 0.29-0.79)$, flare $(r=0.52$, 95\% Cl 0.19-0.75). Uß2M decreased over 1-2 years while SCr returned to normal within a few months.

Conclusions: UB2M correlate with uveitis activity and trend down over the course of TINU. U $2 \mathrm{M}$ may serve as a useful tool in determining where patients are in their systemic disease course.
\end{abstract}

Keywords: $\beta 2$-microglobulin, Tubulointerstitial, Nephritis, Uveitis, TINU

\section{Background}

Tubulointerstitial nephritis and uveitis (TINU) syndrome is a rare, idiopathic inflammatory disorder characterized by acute tubulointerstitial nephritis (TIN) and uveitis without an otherwise known systemic cause [1,2]. Typical ocular involvement is symptomatic, bilateral anterior uveitis [3], though cases of intermediate [4] or panuveitis with chorioretinal lesions [5] have been reported. Conversely, symptoms of TIN are non-specific (e.g., fever, malaise), often mild, and may precede (65\%), follow (21\%), or coincide $(15 \%)$ with the onset of ocular symptoms [2]. TINU syndrome represents only $1-2 \%$ of patients at tertiary uveitis centers [6,7], a likely underrepresentation given the difficulty in recognizing acute TIN without appropriate laboratory testing [8]. TIN in TINU syndrome tends to be self-limited with gradual normalization of renal function,

\footnotetext{
* Correspondence: nasreen-syed@uiowa.edu

'Department of Ophthalmology and Visual Sciences, University of lowa Hospitals and Clinics, 200 Hawkins Drive, lowa City, IA, USA

${ }^{3}$ Iowa City Veterans Affairs Medical Center, 601 Hwy 6 West, lowa City, IA, USA

Full list of author information is available at the end of the article
}

i.e., serum creatinine (SCr). However, uveitis in TINU syndrome is often chronic and relapsing. Improved management of TINU syndrome may minimize sequelae of chronic uveitis and the discomfort associated with symptomatic recurrence of the uveitis.

Definitive diagnosis of TIN requires renal biopsy. However, this invasive procedure carries rare but significant risk of severe or life-threatening hemorrhage. Urinary $\beta 2$-microglobulin (Uß2M), which is elevated in $88-100 \%$ of reported cases of TINU syndrome, has emerged as a useful diagnostic tool in TINU syndrome $[2,8,9]$. The landmark Mandeville diagnostic criteria, detailed in a systematic review by Mandeville, Levinson, and Holland in 2001 [2], incorporated U $32 \mathrm{M}$ as part of evidence for interstitial nephritis. In healthy individuals, $U \beta 2 M$ is a small protein excreted by the renal glomeruli into the ultrafiltrate, with $90 \%$ resorbed by the tubules. In acute TIN, reabsorption of U $\beta 2 M$ is compromised and U $32 \mathrm{M}$ becomes elevated in the urine [10]. In TINU syndrome, $\mathrm{U} \beta 2 \mathrm{M}$ is typically highly elevated and may remain elevated 
for months, long after laboratory tests of renal function [SCr or urinalysis (UA)] have normalized $[2,11]$.

TINU syndrome, with systemic manifestations and laboratory abnormalities, is an inflammatory condition where an indicator of overall disease activity would be valuable in assisting therapeutic decision-making. Though slitlamp findings are typically used to titrate therapy for anterior uveitis, it is difficult to know the activity level of the patient's systemic inflammation and when and if the uveitis will recur after therapy is tapered or discontinued, especially if the eye is quiet on treatment. Traditional markers of systemic inflammation (i.e., sedimentation rate, c-reactive protein) are non-specific and often normal. This study aims to determine whether repeated laboratory analysis of U $\mathrm{U} 2 \mathrm{M}$ levels, or other tests of renal function, correlates with the activity of uveitis over time in TINU syndrome.

\section{Methods}

We conducted a retrospective chart review in the eye clinic at the University of Iowa Hospitals and Clinics from May 1, 2009, to February 15, 2017. This study was approved by the Institutional Review Board, adhered to the tenets of the Declaration of Helsinki, and was compliant with the Health Insurance Portability and Accountability Act.

We selected all patients from the electronic health record (EHR), Epic (Epic Inc., Madison, WI) during the study period with a diagnosis of "iridocyclitis" according to International Classification of Disease codes version 9 (ICD-9) and ICD-10 via an EHR search. ICD-9 codes (Jan. 1, 2009-Oct. 1, 2015) included acute and subacute iridocyclitis, unspecified (364.00), primary iridocyclitis (364.01), recurrent iridocyclitis (364.02), secondary iridocyclitis, noninfectious (364.04), chronic iridocyclitis, unspecified (364.10), unspecified iridocyclitis (364.3). ICD-10 codes (Oct. 1, 2015-Feb. 15, 2017) included primary iridocyclitis (H20.01), unspecified acute and subacute iridocyclitis (H20.00), unspecified iridocyclitis (H20.9), and chronic iridocyclitis (H20.12).

From the patients identified by the EHR search, an EPIC-based laboratory data search for all patients with an elevated U $\beta 2 M(>160 \mu \mathrm{g} / \mathrm{L})$ was performed. From these patients, those with a clinical diagnosis of TINU syndrome made by a uveitis specialist were selected for study. Additional inclusion criteria were follow-up of at least 30 days.

\section{Clinical data}

The following clinical data were collected: initial ocular symptoms, systemic symptoms associated with TINU syndrome, presenting visual acuity (in logarithm of the minimum angle of resolution [logMAR]), intraocular pressure (IOP), uveitis activity (SUN grading [12]), treatment, uveitis relapse or exacerbation, and days of follow-up. Any history of diabetes was noted. An exacerbation was defined as increased inflammation during steroid taper that required escalation of therapy. Relapse was defined as reappearance of anterior chamber inflammation after a minimum of 2 weeks following taper from any topical or systemic immunomodulatory medications. Remission was defined as inactive disease for at least 3 months after discontinuation of all treatment [12].

\section{Laboratory data}

Antinuclear antibody (ANA), cytoplasmic and perinuclear anti-neutrophil cytoplasmic antibody (c- and p-ANCA), angiotensin-converting enzyme (ACE), rheumatoid factor (RF), syphilis serology, Lyme disease serology, HLA-B27, $\mathrm{U} \beta 2 \mathrm{M}, \mathrm{SCr}$, urinalysis (UA), and any renal biopsy results were collected. U $\beta 2 M$ levels greater than $160 \mu \mathrm{g} / \mathrm{L}$ were considered elevated. The initial U $\beta 2 \mathrm{M}$ and any subsequent $\mathrm{U} \beta 2 \mathrm{M}$ measurements were collected. The U $\beta 2 \mathrm{M}$ elevation at each time point was calculated as a ratio of the U $32 \mathrm{M}$ level at that time point to the maximum U $32 \mathrm{M}$ recorded for that patient and expressed as a percentage. $\mathrm{SCr}$ was considered elevated if $>0.9 \mathrm{mg} / \mathrm{dL}$ in patients less than 16 years of age. For males and females older than 16, $\mathrm{SCr}$ was elevated if over 1.2 and $1.0 \mathrm{mg} / \mathrm{dL}$, respectively. Patients were categorized based on Mandeville criteria [2].

\section{Data analysis}

Pearson correlation was calculated between U $\beta 2 \mathrm{M}$ elevation (\%) and uveitis activity (anterior chamber cell, flare, and anterior vitritis) and $\mathrm{SCr}$ and uveitis activity. Statistical analysis was by Prism software (version 7.0a, GraphPad, La Jolla, CA). A 95\% confidence interval $(95 \% \mathrm{CI})$ was calculated to accompany mean data.

\section{Results}

The EHR diagnosis search resulted in 1283 patients. Of these patients, 30/1283 had an elevated U $32 \mathrm{M}$. Two of 30 were excluded for error in diagnosis code (no chart documentation of uveitis), and 7/30 did not meet followup criteria. Of the remaining $21 / 30$ patients, $12 / 21$ were excluded for a probable alternative cause of their uveitis or elevated U $32 \mathrm{M}$ : herpetic iridocyclitis (3), +HLA-B27 (3), + RF (2), highly elevated ACE (1), history of chronic kidney disease (1), IgA nephropathy (1), and history of recurrent/recent kidney stones (1). The remaining $9 / 30$ patients had a clinical diagnosis of TINU syndrome and met criteria for full analysis.

\section{Demographics and clinical characteristics at presentation}

The mean age at diagnosis was 33.4 years (range 12-67, $95 \%$ CI $15.8-51.1$ ) and $56 \%$ of patients were female. All patients had ocular symptoms (Table 1); $33 \%$ had systemic symptoms. Mean $\log$ MAR visual acuity was 0.12 
Table 1 Uveitis symptoms, laterality, onset, and location at presentation

\begin{tabular}{ll}
\hline & No. of patients (\%) \\
\hline Symptoms at presentation & $8(89 \%)$ \\
Redness & $7(78 \%)$ \\
Photophobia & $6(67 \%)$ \\
Decreased vision & $6(67 \%)$ \\
Pain & $5(56 \%)$ \\
Tearing & $3(33 \%)$ \\
Floaters & \\
Laterality and onset & $9(100 \%)$ \\
Bilateral & $6(67 \%)$ \\
Sequential involvement & \\
Primary location of uveitis & $6(67 \%)$ \\
Anterior & $2(22 \%)$ \\
Intermediate & $1(11 \%)$ \\
Panuveitis &
\end{tabular}

(95\% CI 0.04-0.21) and mean IOP was $14.6 \mathrm{mmHg}$. All patients ultimately had bilateral uveitis, and $67 \%$ had primarily anterior uveitis (Table 1 ).

\section{Laboratory trends and uveitis activity}

Mean Uß2M at presentation was $6536 \mu \mathrm{g} / \mathrm{L}(95 \% \mathrm{CI}$ 2030-11,043), which was 40.9 times the upper limit of normal (normal $<160 \mu \mathrm{g} / \mathrm{L}$ ). The median interval between the onset of uveitis and initial U $32 \mathrm{M}$ testing was 7 days (mean 26.4 [95\% CI - 12.6-65.5]). There was a strong correlation between U $32 \mathrm{M}$ elevation and anterior chamber cell ( $r=0.69,95 \%$ CI 0.46-0.84) (Fig. 1a) and between Uß2M elevation and flare $(r=0.65$, 95\% CI 0.39-0.81). There was no significant correlation with UB2M and anterior vitreous cell $(r=0.33,95 \%$ CI $-0.12-0.66)$. In seven patients that had repeated $\mathrm{U} \beta 2 \mathrm{M}$ measurements, U $32 \mathrm{M}$ trended downwards over the course of 1-2 years for most patients (Fig. 1c), and the mean final U $32 \mathrm{M}$ was $12.2 \%$ of the initial U $32 \mathrm{M}$ (95\% CI - 2.4-26.8). U $32 \mathrm{M}$ had normalized in four patients at last follow-up. No significant correlation was detected between anterior vitreous cell and Uß2M elevation ( $r=0.32$ 95\% CI - 0.12-0.66).

$\mathrm{SCr}$ was elevated at presentation in $7 / 9$ patients $(78 \%)$ with a mean $\mathrm{SCr}$ of $1.28 \mathrm{mg} / \mathrm{dL}$ (95\% CI 1.00-1.56). SCr had a trend for weaker correlation with anterior chamber cell $(r=0.59,95 \%$ CI 0.29-0.79) (Fig. 1b) and flare $(r=0.52,95 \%$ CI $0.19-0.75)$ compared to U $\beta 2 \mathrm{M}$, but the difference was not significant. There was no significant correlation with $\mathrm{SCr}$ and anterior vitreous cell $(r=0.21$, 95\% CI - 0.28-0.62). Over the disease course, $\mathrm{SCr}$ returned to normal within a few months and varied little after that time (Fig. 1d). SCr normalized in 6/7 patients. Mean final SCr was $0.93 \mathrm{mg} / \mathrm{dL}$ (95\% CI 0.80-1.07).
Urine protein was detected on UA in just five patients: trace protein in four patients $(44 \%)$ and $1+$ protein in one patient (11\%). No patient required dialysis. Table 2 shows the systemic laboratory workup and Mandeville criteria for individual patients. A renal biopsy was performed in three patients; all biopsies showed acute TIN. Four patients met Mandeville criteria for definitive TINU syndrome; five were probable. One patient had a history of gestational diabetes (Table 2, patient 6), which had resolved, but none of the other patients were diabetic.

\section{Treatment, follow-up, and ocular complications}

All patients were treated with topical steroids, and oral steroids were used in $8 / 9$ patients with a mean starting dose of $0.6 \mathrm{mg} / \mathrm{kg} /$ day (95\% CI $0.5-0.8$ ). Two patients (patients 1 and 2, Table 2) were also treated with mycophenolate mofetil by nephrology. Mean follow-up was 36.2 months (95\% CI 15.6-56.9). At last follow-up, five (56\%) patients were in remission. Relapse occurred once in two different patients. An exacerbation occurred in 7/ 9 patients within the first year. Patients had a mean final - $0.01 \log$ MAR visual acuity (95\% CI - 0.03-0.02), which was a significant improvement from presentation. Ocular complications are shown in Table 3.

\section{Conclusions}

TINU syndrome represents $1-2 \%$ of patients at tertiary uveitis centers $[6,7]$, which is a likely underrepresentation given the difficulty in recognizing acute TIN without appropriate laboratory testing early in the course of the condition [8]. Acute renal dysfunction (elevated SCr) may be self-limited in TINU syndrome, and traditional laboratory tests of renal function have been shown to rapidly normalize in TINU patients [11]. Depending on the timing of uveitis presentation, which precedes or follows TIN in $86 \%$ of patients, SCr may be normal and is not a useful marker for any residual, subclinical systemic disease [2]. Diagnosis represents only the first of many hurdles in the management of TINU syndrome, as uveitis tends to be chronic and relapsing, potentially leading to ocular sequelae. Most patients eventually end up with remission of uveitis, though the timing of remission varies $[8,13]$.

Multiple authors have proposed U $\beta 2 \mathrm{M}$ as a potentially useful diagnostic laboratory test in TINU syndrome $[2,8,9$, 13, 14]. In 1999, Takemura and colleagues found that Uß2M was highly elevated in a cohort of patients with biopsy-proven TINU syndrome. U $\beta 2 \mathrm{M}$ decreased gradually over the course of the disease, remaining elevated longer than SCr. Mandeville then incorporated U $\beta 2 \mathrm{M}$ into the diagnostic criteria for interstitial nephritis and abnormal urinalysis, recommending testing if $\mathrm{SCr}$ was abnormal. Goda et al. later demonstrated that $92 \%$ of biopsy-proven TINU patients had elevated U $\beta 2 \mathrm{M}$, while SCr, a marker of acute renal dysfunction, was elevated in just 25\% [14]. In 


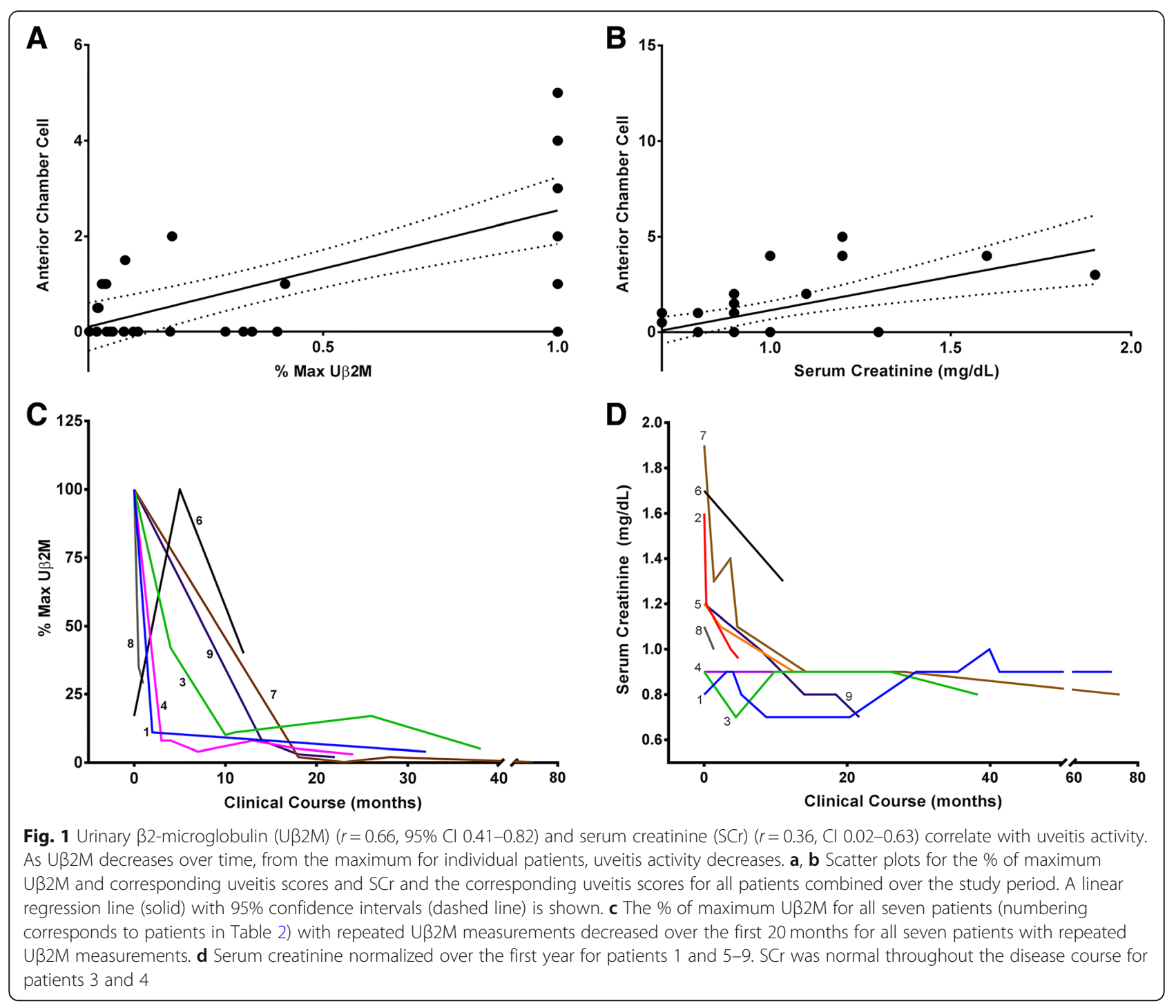

2015, Hettinga et al. brought U $\beta 2 \mathrm{M}$ to the forefront as a diagnostic tool by demonstrating that $\mathrm{U} \beta 2 \mathrm{M}$ has a positive predictive value (PPV) of $88 \%$ and a negative predictive value of $97 \%$ in detecting definite or probable TINU. When combined with in elevated SCr, the PPV increases to $100 \%$ [9].

We hypothesized that the role of U $\beta 2 \mathrm{M}$ may not be limited to that of a diagnostic test but may also provide information on the course of inflammatory activity in TINU syndrome. Our study is the first to report that U $32 \mathrm{M}$ levels correlate strongly with the activity of ocular inflammation over the course of TINU syndrome, and this correlation tends to be stronger than that of $\mathrm{SCr}$. Whereas SCr rapidly normalizes, our cohort showed a decrease in U $32 \mathrm{M}$ over the course of their disease, which is similar to previously published data [11]. These data suggest that $U \beta 2 M$ levels may guide the clinician, over time, in determining where the patient is in the disease course, ultimately helping with treatment decisions. U $32 \mathrm{M}$ levels may serve as an adjunct to observation of uveitis activity at the slit lamp in regard to when treatment for the uveitis may be discontinued. When the U $\beta 2 M$ levels have normalized, our study suggests that the chance of the uveitis recurring after treatment is discontinued is far less than if the U $32 \mathrm{M}$ is still elevated. This is a unique finding in inflammatory ocular disease since the ability to anticipate recurrence is difficult upon stopping treatment in most forms of uveitis.

Our results also confirm that U $32 \mathrm{M}$ and $\mathrm{SCr}$ do not covary much in patients with TINU syndrome, which is in accordance with findings in biopsy-proven TINU syndrome $[11,14]$. U $32 \mathrm{M}$ is passed almost completely through the glomerular membrane and then almost entirely reabsorbed in the normal proximal tubule. Thus, $\mathrm{U} \beta 2 \mathrm{M}$ elevation in disease is primarily determined by proximal tubular dysfunction and is a marker for TIN 


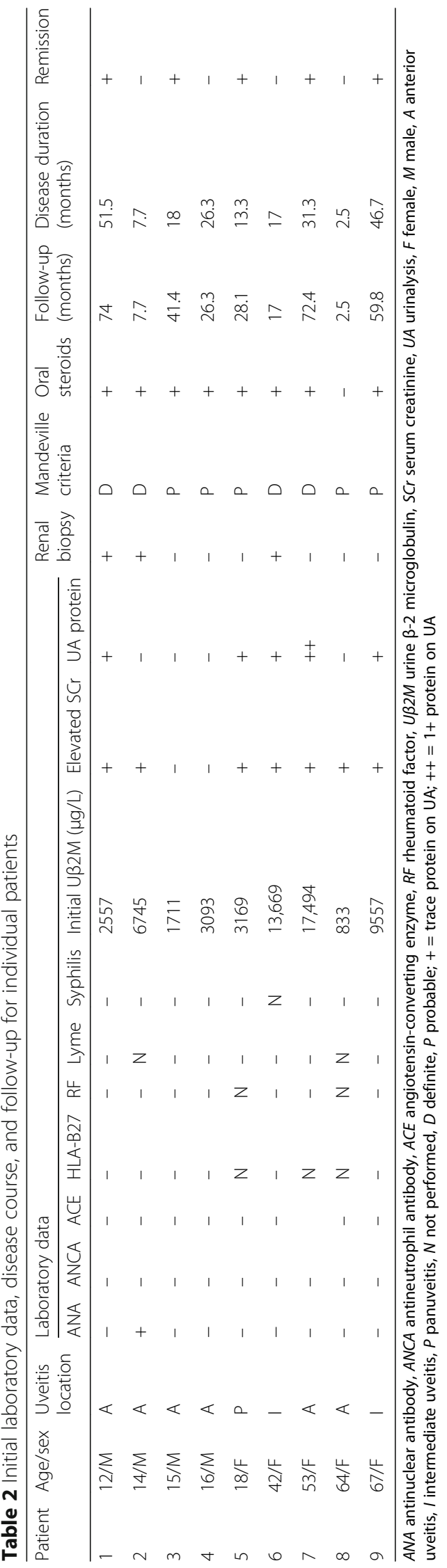


Table 3 Ocular complications throughout the disease course

\begin{tabular}{lll}
\hline & Patients & Resolved \\
\hline Posterior synechiae & 1 & $1 / 1$ \\
Cataract & 2 & $2 / 2$ \\
OHT/glaucoma & $3^{\mathrm{a}}$ & $1 / 3$ \\
Cystoid macular edema & 1 & $1 / 1$ \\
\hline
\end{tabular}

OHT Ocular hypertension

${ }^{a}$ One patient had preexisting glaucoma

[15]. Conversely, SCr is filtrated by the glomeruli as well as actively secreted by the proximal tubule and is considered a marker for nephropathy [16]. In TINU syndrome, the fact that $\mathrm{U} \beta 2 \mathrm{M}$ can be elevated while $\mathrm{SCr}$ is normal, as well as the stronger correlation of $\mathrm{U} \beta 2 \mathrm{M}$ elevation with uveitis activity, together seems to indicate that $\mathrm{U} \beta 2 \mathrm{M}$ may be a more specific activity marker for ongoing subclinical systemic inflammation [15].

While our retrospective study makes a significant contribution to the small pool of literature on TINU syndrome, it has limitations. The study group was relatively small due to the rarity of the disease, and not all patients had complete data based on the retrospective nature of the study. Follow-up was variable due to the retrospective design of the study, which limits our understanding of relapse and remission. U $32 \mathrm{M}$ testing, when repeated, was done rarely and at variable time points. Data is insufficient to detect whether U $32 \mathrm{M}$ may predict relapse, treatment requirement, or complications.

In summary, we describe the novel finding of $U \beta 2 M$ correlation with uveitis activity in TINU syndrome, implicating $\mathrm{U} \beta 2 \mathrm{M}$ as an objective marker of systemic inflammatory activity and uveitis activity in patients with TINU syndrome. U $32 \mathrm{M}$, especially in the setting of clinically inactive uveitis treatment, may provide information on where a patient is in their disease course and when a patient may safely taper or discontinue therapy. Additional study with prospective and more uniform intervals of U $32 \mathrm{M}$ measurement is recommended to further investigate the role of $\mathrm{U} \beta 2 \mathrm{M}$ in TINU syndrome.

\section{Abbreviations \\ AC: Anterior chamber; ACE: Angiotensin-converting enzyme; ANA: Antinuclear antibody; ANCA: Anti-neutrophil antibody; Cl: Confidence interval; EHR: Electronic health record; ICD: International Classification of Disease; IOP: Intraocular pressure; RF: Rheumatoid factor; SCr: Serum creatinine; SUN: Standardized uveitis nomenclature; TIN: Tubulointerstitial nephritis; TINU: Tubulointerstitial nephritis and uveitis; UA: Urinalysis; U $2 \mathrm{M}$ : Urinary $\beta 2$-microglobulin}

\section{Acknowledgements}

Not applicable.

\section{Funding}

This work was supported by unrestricted Funds from Research to Prevent Blindness, New York, NY. Michael D. Abràmoff is supported by the Robert C Watzke Professorship of Ophthalmology and Visual Sciences, lowa City, IA.

\section{Availability of data and materials}

The data generated during and/or analyzed during the current study are available from the corresponding author upon reasonable request.

\section{Authors' contributions \\ LMP and AMF carried out data collection and analysis. LMP participated in the study and design, coordinated the study, and drafted the manuscript. MDA helped with statistical analysis and study design. NAS conceived the study and participated in the design, along with editing of the manuscript.} All authors read and approved the final manuscript.

\section{Ethics approval and consent to participate}

This study was approved by the University of lowa Institutional Review Board, adhered to the tenets of the Declaration of Helsinki, and was compliant with the Health Insurance Portability and Accountability Act. Waiver of documentation of consent and waiver of elements of consent were granted due to the retrospective nature of the study.

\section{Consent for publication}

Identifiable individual patient data was not included in the study and therefore consent to publish from individual patients was not obtained or required.

\section{Competing interests}

The authors declare that they have no competing interests related to this work. MDA has the following financial interests and consultancy: IDx LLC, lowa City, IA, founder; IDx LLC, lowa City, IA, investor (equity interests); IDx LLC, lowa City, IA, consultant; IDx LLC, lowa City, IA, president; Gerson Lehrman Group, consultant, expert testimony; iOptics BV, Amsterdam Netherlands, founder; iOptics BV, Amsterdam Netherlands, investor (equity interests); EASDEC invited speaker; EU retina invited speaker; ATA invited speaker; and MICCAI invited speaker. Dr. Abràmoff has the following intellectual property leading to royalties: University of lowa patents, University of lowa patent applications, IDx LLC patents, IDx LLC patent applications.

\section{Publisher's Note}

Springer Nature remains neutral with regard to jurisdictional claims in published maps and institutional affiliations.

\section{Author details}

${ }^{1}$ Department of Ophthalmology and Visual Sciences, University of lowa Hospitals and Clinics, 200 Hawkins Drive, lowa City, IA, USA. ${ }^{2}$ The Stephen A. Wynn Institute for Vision Research, University of lowa, 375 Newton Road, 4111 MERF, lowa City, IA, USA. ${ }^{3}$ lowa City Veterans Affairs Medical Center, 601 Hwy 6 West, lowa City, IA, USA. ${ }^{4}$ Department of Pathology, University of lowa Hospitals and Clinics, 200 Hawkins Drive, lowa City, IA, USA.

Received: 30 April 2018 Accepted: 12 December 2018

Published online: 29 December 2018

\section{References}

1. Dobrin RS, Vernier RL, Fish AL (1975) Acute eosinophilic interstitial nephritis and renal failure with bone marrow-lymph node granulomas and anterior uveitis. A new syndrome. Am J Med 59(3):325-333

2. Mandeville JT, Levinson RD, Holland GN (2001) The tubulointerstitial nephritis and uveitis syndrome. Surv Ophthalmol 46(3):195-208

3. Mackensen F, Billing H (2009) Tubulointerstitial nephritis and uveitis syndrome. Curr Opin Ophthalmol 20(6):525-531

4. Legendre M, Devilliers H, Perard L et al (2016) Clinicopathologic characteristics, treatment, and outcomes of tubulointerstitial nephritis and uveitis syndrome in adults: a national retrospective strobe-compliant study. Medicine (Baltimore) 95(26):e3964

5. Reddy AK, Hwang YS, Mandelcorn ED, Davis JL (2014) HLA-DR, DQ class II DNA typing in pediatric panuveitis and tubulointerstitial nephritis and uveitis. Am J Ophthalmol 157(3):678-686 e671-672

6. Kump LI, Cervantes-Castaneda RA, Androudi SN, Foster CS (2005) Analysis of pediatric uveitis cases at a tertiary referral center. Ophthalmology 112(7): 1287-1292

7. Rosenbaum JT (1988) Bilateral anterior uveitis and interstitial nephritis. Am J Ophthalmol 105(5):534-537 
8. Mackensen F, Smith JR, Rosenbaum JT (2007) Enhanced recognition, treatment, and prognosis of tubulointerstitial nephritis and uveitis syndrome. Ophthalmology 114(5):995-999

9. Hettinga YM, Scheerlinck LM, Lilien MR, Rothova A, de Boer JH (2015) The value of measuring urinary beta2-microglobulin and serum creatinine for detecting tubulointerstitial nephritis and uveitis syndrome in young patients with uveitis. JAMA Ophthalmol 133(2):140-145

10. Karlsson FA, Wibell L, Evrin PE (1980) beta 2-Microglobulin in clinical medicine. Scand I Clin Lab Invest Suppl 154:27-37

11. Takemura T, Okada M, Hino S et al (1999) Course and outcome of tubulointerstitial nephritis and uveitis syndrome. Am J Kidney Dis 34(6): 1016-1021

12. Jabs DA, Nussenblatt RB, Rosenbaum JT (2005) Standardization of uveitis nomenclature working G. Standardization of uveitis nomenclature for reporting clinical data. Results of the first international workshop. Am J Ophthalmol 140(3):509-516

13. Sobolewska B, Bayyoud T, Deuter C, Doycheva D, Zierhut M (2016) Longterm follow-up of patients with tubulointerstitial nephritis and uveitis (TINU) syndrome. Ocul Immunol Inflamm 12:1-7

14. Goda C, Kotake S, Ichiishi A, Namba K, Kitaichi N, Ohno S (2005) Clinical features in tubulointerstitial nephritis and uveitis (TINU) syndrome. Am J Ophthalmol 140(4):637-641

15. Wibell $L$ (1978) The serum level and urinary excretion of beta2microglobulin in health and renal disease. Pathol Biol (Paris) 26(6):295-30

16. Perrone RD, Madias NE, Levey AS (1992) Serum creatinine as an index of renal function: new insights into old concepts. Clin Chem 38(10):1933-1953

\section{Submit your manuscript to a SpringerOpen ${ }^{\circ}$ journal and benefit from:}

- Convenient online submission

- Rigorous peer review

- Open access: articles freely available online

- High visibility within the field

- Retaining the copyright to your article

Submit your next manuscript at $\boldsymbol{\nabla}$ springeropen.com 